\title{
Anisotropic Light Scattering from the Rigid Structure of DNA
}

\author{
J. García de la Torre and Arturo Horta \\ Departmento de Química Física, Facultad de Química, \\ Universidad Complutense, Madrid-3, Spain.
}

(Received July 7, 1976)

\begin{abstract}
The theory of anisotropic light scattering developed by Nagai is valid for any macromolecular structure. It is applied here to the calculation of polarized light scattering from DNA in a completely rigid configuration. This configuration is the one adopted by DNA fragment molecules of low molecular weight. The polarized light-scattering characteristics of such a rigid configuration are adequately represented by the model of an infinitely thin rod with two polarizabilities. The influence of the double helical structure of finite thickness is vanishingly small. The rod model is used to establish the range of validity of Nagai's approximate formulas. This range is extremely limited; for DNA fragments: $\theta \leq 10^{\circ}$. It is suggested that the best analysis of light-scattering data, whether polarized or unpolarized, is to use the exact formulas of the rod model, instead of looking for limiting laws valid either at low or high angles.

KEY WORDS Light Scattering / Optical Anisotropy / Nagai's Theory / DNA Molecule / Double Helix / Rigid Rod Model / Zimm Plot /
\end{abstract}

A general theory of elastic (Rayleigh-Debye) light scattering by an isotropic system composed of anisotropic units has been recently developed by Nagai. ${ }^{1,2}$ It is useful for the calculation of anisotropic light scattering from macromolecules in solution.

The units which are included in polymer chains are always anisotropic and their optical properties have to be represented by a polarizability tensor having three principal components. Prior to Nagai's contribution, several theories were developed to describe light scattering from polymer chains composed of anisotropic units. For example, Utiyama and $\mathrm{Kurata}^{3,4}$ studied the random coil, and Horn, et al., ${ }^{5,6}$ studied the rigid rod (with unequal longitudinal and transversal polarizabilities). These theories are particular results derived for special models. The theory of Nagai is, on the other hand, general. It does not contain any assumption about the distribution of units in the chain and is, thus, valid for polymers of any structure.

Formally, the theory of Nagai is exact. However, it yields formulas that are too complex for detailed numerical calculations. To circumvent this difficulty and to be able to obtain results that could be compared with experiment, Nagai expanded the anisotropic part of the scattered intensities in powers of the difference between incident and scattered wavevectors. ${ }^{1}$ The first few terms in this expansion are still complex but amenable to practical calculations. The corresponding results are only approximate because of the neglect of higher order terms.

Nagai applied this approximate theory to the wormlike-chain model, ${ }^{1,2}$ and Patterson to the case of realistic chains of polymethylene. ${ }^{7}$ However, as Patterson pointed out in his paper, the importance of anisotropic light scattering should be largest when "...the chains are not in a random-coil state but adopt a regular repeating conformation such as a helix..."." The case of the DNA molecule is, then, a typical example of a macromolecule for which the theory of Nagai should be more relevant, because its molecular structure is that of a double helix which shows a measurable negative anisotropy.

In this paper we study the application of 


\section{J. G. de la Torre and A. Horta}

Nagai's theory to the light scattered by DNA. Only rigid conformations are considered. Empirically, this corresponds to short fragment molecules of DNA (molecular weights up to about $5 \times 10^{5}$ ).

Our first point in this paper is to determine the range of validity of Nagai's approximate equations in the case of the DNA molecule. The most direct way for determining this range is to compare the theoretical intensities which are calculated with the exact and approximate equations. For the real structure of DNA this is not possible because, as explained above, the exact formulas of Nagai are not useful. However, for the simplified model of a rigid rod, the exact results are known and have been given by Horn, et al. ${ }^{5,6}$ It is, then, possible to establish the range of validity of Nagai's approximate equations for the case of such a model of DNA. That is what we discuss first in this paper.

The rod model is the one usually applied to interpret the optical anisotropy of DNA..$^{8-11}$ However, the representation of the molecule as an infinitely thin rod with two principal polarizabilities is an idealization of the DNA structure which neglects its finite cross-section and its helical character.

Our second point of discussion in this paper is to determine what is the importance of the double helical structure of DNA for its anisotropic light scattering. This can be studied thanks to the theory of Nagai, which is valid for any macromolecular configuration. As a representation of the real structure of DNA we use here a double helical model recently proposed by us for the study of X-ray diagrams and hydrodynamic properties. $^{12}$ By using Nagai's approximate equations we calculate the lightscattering behavior of such a double helical model and compare the corresponding results with the ones obtained for the idealized rod of zero thickness.

\section{MODELS FOR DNA}

In our double helical model ${ }^{12}$ the scattering units are placed along a double helix of radius $A$ and pitch $34 \AA$. There are 20 units per turn so that a one to one correspondence exists between chain units and nucleotides of the DNA molecule. We arbitrarily choose the axis of the double helix as the $z$-axis of a fixed cartesian frame of reference. The coordinates of any scattering unit, $i$, referred to such a frame, are given by ${ }^{12}$ :

$$
\begin{aligned}
& x_{i}=A \cos \left(t_{i}+\omega\right) \\
& y_{i}=A \sin \left(t_{i}+\omega\right) \\
& z_{i}=(34 / 2 \pi) t_{i}
\end{aligned}
$$

where

$$
t_{i}=0.2 \pi \text { int }\{(i-1) / 2\}
$$

and int ( ) means integer part. In one of the two helices $\omega=0$, while in the other $\omega=\pi$.

We represent the anisotropy of the units by means of three different principal polarizabilities, $\alpha_{1}, \alpha_{2}$, and $\alpha_{3}$, having the following directions: $\alpha_{1}$ is tangent to the helix and is intended to represent the polarizability along the direction defined by the sugar-phosphate sequence; $\alpha_{2}$ is perpendicular to the helical axis and it should represent the intense polarizability of the bases ${ }^{13}$; $\alpha_{3}$ is perpendicular to the plane defined by $\alpha_{1}$ and $\alpha_{2}$. Simple geometric relationships yield for unit $i$ the following polarizability tensor, $\gamma_{i}$, expressed in the frame of reference fixed to the helix:

$$
\begin{aligned}
\gamma_{i}= & \left(\begin{array}{ccc}
-c_{1} s & c & c_{2} s \\
c_{1} c & s & -c_{2} c \\
c_{2} & 0 & c_{1}
\end{array}\right)\left(\begin{array}{ccc}
\alpha_{1} & 0 & 0 \\
0 & \alpha_{2} & 0 \\
0 & 0 & \alpha_{3}
\end{array}\right) \\
& \times\left(\begin{array}{ccc}
-c_{1} s & c_{1} c & c_{2} \\
c & s & 0 \\
c_{2} s & -c_{2} c & c_{1}
\end{array}\right)
\end{aligned}
$$

where the symbols $s, c, c_{1}$, and $c_{2}$, stand for

$$
\left.\begin{array}{rl}
s & =\sin t_{i} \\
c & =\cos t_{i} \\
c_{1} & =\left\{1+(3.4 / 2 \pi A)^{2}\right\}^{-1 / 2} \\
c_{2} & =\left\{1+(2 \pi A / 3.4)^{2}\right\}^{-1 / 2}
\end{array}\right\}
$$

The more simple model of a rigid rod, calculated by Horn, et al., ${ }^{5,6}$ has only two principal values of the polarizability: one along the rod direction, $\alpha_{\|}$, and another (degenerate) perpendicular to it, $\alpha_{\perp}$. The anisotropy can be characterized in this model by means of a parameter $\delta$, defined as 


$$
\delta=\frac{\alpha_{\|}-\alpha_{\perp}}{2 \alpha_{\perp}+\alpha_{\|}}
$$

Different experimental studies of polarized light scattering ${ }^{5-9}$ and of flow birefringence ${ }^{14,15}$ yield for DNA values of $\delta$ in between -0.10 and -0.15 .

It is easy to establish a correspondence between the double helical model and the rod model. When the polarizability tensors of the units composing the double helical model are averaged over an integer number of turns, the resulting tensor is diagonal and has the same value for the two polarizabilities perpendicular to the $z$-axis. This is equivalent to an anisotropic rod having principal components of the polarizability, $\alpha_{\|}{ }^{\prime}$ and $\alpha_{\perp}{ }^{\prime}$, related to $\alpha_{1}, \alpha_{2}$, and $\alpha_{3}$, by

$$
\begin{aligned}
& \alpha_{\|}{ }^{\prime}=c_{2}{ }^{2} \alpha_{1}+c_{1}{ }^{2} \alpha_{3} \\
& \alpha_{\perp}{ }^{\prime}=\left(c_{1}^{2} \alpha_{1}+\alpha_{2}+c_{2}^{2} \alpha_{3}\right) / 2
\end{aligned}
$$

\section{LIGHT-SCATTERING INTENSITIES}

According to Nagai's theory, ${ }^{1}$ the Rayleigh ratio, $R$, for different states of polarization ( $\mathrm{V} \equiv$ vertical, $\mathrm{H} \equiv$ horizontal), is given by

$$
\begin{aligned}
R_{\mathrm{VV}} / K c M= & (N \bar{\gamma})^{-2}\left\{f_{2}-f_{3} h^{2}+\cdots+(N \bar{\gamma})^{2} P(\theta)\right\} \quad(10) \\
R_{\mathrm{VH}} / K c M= & (N \bar{\gamma})^{-2}\left\{\frac{3}{4} f_{2}-f_{4} h^{2}-f_{5} h^{2} \cos \theta+\cdots\right\}(11) \\
R_{\mathrm{HH}} / K c M= & (N \bar{\gamma})^{-2}\left\{\frac{3}{4} f_{2}-f_{6} h^{2}-f_{7} h^{2} \cos \theta+\cdots\right. \\
& \left.+\left[\frac{1}{4} f_{2}-f_{8} h^{2}+\cdots+(N \bar{\gamma})^{2} P(\theta)\right] \cos ^{2} \theta\right\}
\end{aligned}
$$

The first letter in the subscript of $R$ denotes the polarization of the incident light and the second letter the polarization of the scattered light. $h$ is a function of the scattering angle, $\theta$, and of the wavelength of the light, $\lambda$ :

$$
h=\frac{4 \pi}{\lambda} \sin \frac{\theta}{2}
$$

In eq $10-12$, the anisotropic contribution to $R$ is considered up to terms of order $h^{2} . N$ is the number of scattering units, and $\bar{\gamma}$ the mean polarizability of any unit:

$$
\bar{\gamma}=\frac{1}{3} \operatorname{Tr} \gamma_{i i}
$$

$K, c$, and $M$, have their usual meanings of optical constant, concentration, and molecular weight, respectively.

$f_{2}, f_{3}, \ldots, f_{7}$, are quantities independent of angle and defined in eq 55-61 of ref 1 . They can be written in a more compact way as follows:

$$
\begin{gathered}
f_{2}=\frac{2}{15} \sum_{i=1}^{N} \sum_{j=1}^{N} \operatorname{Tr} \hat{\gamma}_{i} \hat{\gamma}_{j} \\
\frac{1}{2} f_{3}, f_{7}, f_{8}=(N \bar{\gamma})^{2}\left(x_{1} V_{1}+x_{2} V_{2}+x_{3} V_{3}\right) / 210 \\
f_{4}, f_{5}, f_{6}=\left(N_{\bar{\gamma}}\right)^{2}\left(x_{4} V_{2}+x_{5} V_{3}\right) / 210
\end{gathered}
$$

where

$$
\hat{\boldsymbol{\gamma}}_{i}=\boldsymbol{\gamma}_{i}-\bar{\gamma} \mathbf{E}
$$

( $\mathbf{E} \equiv$ unit tensor of rank 3$) . \quad{ }^{4} x_{1}, x_{2}, \ldots, x_{5}$, are numerical coefficients, which have different value for each $f$; they are shown explicitly in the Appendix. $V_{1}, V_{2}, V_{3}$, are quantities which represent the combined influence of the geometry of the chain and of the anisotropy of its units. They are given by

$$
\begin{aligned}
& V_{1}=(N \bar{\gamma})^{-2} \bar{\gamma} \sum_{i=2}^{N} \sum_{j=1}^{N-1} \boldsymbol{r}_{i j}^{\mathrm{T}}\left(\hat{\gamma}_{i}+\hat{\gamma}_{j}\right) \boldsymbol{r}_{i j} \\
& V_{2}=(N \bar{\gamma})^{-2} \sum_{i=2}^{N} \sum_{j=1}^{N-1} r_{i j}^{2} \operatorname{Tr} \hat{\gamma}_{i} \hat{\gamma}_{j} \\
& V_{3}=(N \bar{\gamma})^{-2} \sum_{i=2}^{N} \sum_{j=1}^{N-1} \boldsymbol{r}_{i j}^{\mathrm{T}} \hat{\gamma}_{i} \hat{\gamma}_{j} \boldsymbol{r}_{i j}
\end{aligned}
$$

where $\boldsymbol{r}_{i j}$ means the vector distance (column vector) between units $i$ and $j$, and the superscript $\mathrm{T}$ denotes transpose (row vector). These $V$ 's are closely related to what Patterson calls the invariants $\langle Q\rangle$ in his application of Nagai's theory $^{7}$ (actually, $\left(N_{\bar{\gamma}}\right)^{2} V_{1}=\left\langle Q_{2}\right\rangle+\left\langle Q_{3}\right\rangle,\left(N_{\bar{\gamma}}\right)^{2} V_{2}=$ $\left\langle Q_{4}\right\rangle$, and $\left.\left(N_{\bar{\gamma}}\right)^{2} V_{3}=\left\langle Q_{5}\right\rangle\right)$.

$P(\theta)$, in eq 10 and 12 , is the form factor for the macromolecule, which is given by

$$
P(\theta)=\frac{1}{N^{2}} \sum_{i=1}^{N} \sum_{j=1}^{N} \frac{\sin h r_{i j}}{h r_{i j}}
$$

and constitutes the isotropic contribution to $R$. Since we consider a rigid structure for DNA, no configurational average of eq 22 is required.

We first study in this paper the range of validity of the above equations for $R$. To this end, we apply them to the model of a rigid rod of vanishing cross-section. The exact expressions of $R$ for this model are known and have been given by Horn, et al. ${ }^{5,6}$ (eq $1-3$ in ref 5). In these author's notation, $V_{v}$ corresponds to our 


\section{J. G. de la Torre and A. Horta}

$R_{\mathrm{VV}} / K c M, \delta$ has the same meaning as here, and $x=h L / 2$, where $L$ is the total length of the rod. If the spacing between scattering units along the rod is small compared to its total length, $L$, then Nagai's theory yields for the rod model:

$$
\begin{aligned}
& (N \bar{\gamma})^{-2} f_{2}=4 \delta^{2} / 5 \\
& V_{1}=L^{2} \delta / 3 \\
& V_{2}=L^{2} \delta^{2} / 2 \\
& V_{3}=L^{2} \delta^{2} / 3
\end{aligned}
$$

We use eq $10-12,15-17,19-21$, and $23-26$, and take the result of $P(\theta)$ :

$$
P(\theta)=\frac{2 \operatorname{Si}(h L)}{h L}-\frac{4 \sin ^{2}(h L / 2)}{(h L)^{2}}
$$

$\left(\mathrm{Si}(x)=\int_{0}^{x} t^{-1} \sin t \mathrm{~d} t\right)$, to evaluate the $R$ 's according to Nagai's theory for the rigid rod. We then compare our results with the ones obtained

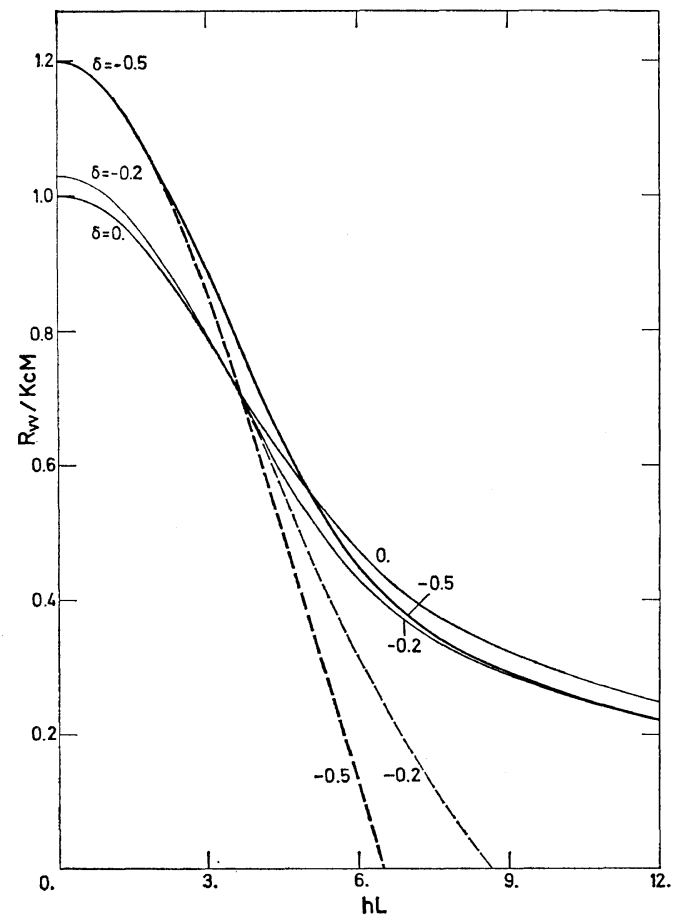

Figure 1. Rayleigh ratios for polarized light scattering from a rigid rod, as a function of the angular variable, $h$, and rod length, $L$, for different values of the anisotropy, $\delta$ : —, exact results calculated according to Horn, et al. 5,6 ; --_, approximate results calculated according to Nagai. ${ }^{1}$ using the exact formulas of Horn, et al. ${ }^{5,6}$

This comparison is show on Figure 1 for $R_{\mathrm{VV}}$. It is represented $v s$. $h L$ for different values of the anisotropy parameter, $\delta$. The exact results are drawn as continuous lines and the approximate ones, from Nagai's theory, as discontinuous lines. The same comparison for $R_{\mathrm{VH}}$ and $R_{\mathrm{HH}}$ is not so easily drawn because they depend, not only on $h L$, but also on $\cos \theta$ (see eq 11 and 12). However, the conclusions that are obtained regarding the angular range for which the approximate formulas are valid are the same as for $R_{\nabla \nabla}$, so that Figure 1 serves as a good example of the three polarized components.

As a second point in this paper, we study the influence of the finite, helical cross-section of DNA structure on the $R$ values. This we do by comparing the scattering behavior of our double helical model (described above) with that of the infinitely thin rod. For the double helix, we calculate $f_{2}, V_{1}, V_{2}$, and $V_{3}$, from the polarizability tensor (eq 5) and cartesian coordinates (eq 1-3) of the units. Actually, there is no need to calculate the $R$ 's in detail, because the differences between the rod and the double helix

Table I. Comparison between the double helical model (unprimed symbols) and the rigid rod model (primed symbols), as a function of the number of scattering units in the macromolecule, $N$. The quantities $f_{2}, V_{1}, V_{2}, V_{3}$, are defined in eq 15 and 19-21. They have been calculated with $A=8 \AA$, $\alpha_{1}=90 \AA^{3}, \alpha_{2}=40 \AA^{3}, \alpha_{3}=20 \AA^{3}, \alpha_{\|^{\prime}}=20.32 \AA^{3}, \alpha_{\perp}^{\prime}=$ $64.84 \AA^{3}$.

\begin{tabular}{rccrc}
\hline$N$ & $\frac{(N \bar{\gamma})^{-2} f_{2}}{\left(N^{\prime} \bar{\gamma}^{\prime}\right)^{-2} f_{2^{\prime}}}$ & $\frac{V_{1}}{V_{1^{\prime}}}$ & $\frac{V_{2}}{V_{2^{\prime}}}$ & $\frac{V_{3}}{V_{3^{\prime}}}$ \\
\hline 6 & 1.271 & 2.668 & 21.947 & 1.280 \\
10 & 0.999 & 1.161 & 5.076 & 0.827 \\
16 & 1.038 & 1.198 & 2.754 & 1.093 \\
20 & 1.000 & 1.155 & 1.928 & 1.089 \\
26 & 1.014 & 1.132 & 1.663 & 1.096 \\
30 & 1.000 & 1.115 & 1.434 & 1.088 \\
40 & 1.000 & 1.090 & 1.263 & 1.075 \\
50 & 1.000 & 1.074 & 1.182 & 1.064 \\
60 & 1.000 & 1.062 & 1.137 & 1.056 \\
80 & 1.000 & 1.047 & 1.088 & 1.044 \\
100 & 1.000 & 1.038 & 1.064 & 1.036 \\
130 & 1.000 & 1.029 & 1.045 & 1.028 \\
160 & 1.000 & 1.024 & 1.034 & 1.023 \\
200 & 1.000 & 1.019 & 1.025 & 1.019 \\
\hline
\end{tabular}


are already contained in the values of $f_{2}, V_{1}, V_{2}$, and $V_{3}$. Hence, it is enough to compare the values calculated for those quantities according to both model chains.

The results obtained from this comparison are shown on Table I, where the primed symbols refer to the rod and the unprimed ones to the double helix. The length of the equivalent rod is taken equal to the maximum $z$-value of the double helix in this calculation.

\section{DISCUSSION}

The analysis of the data represented on Figure 1 shows that the approximate equations of Nagai have a very limited range of validity for the rigid rod. For example, for a DNA fragment of $L=1500 \AA$ (equivalent to $M=3 \times 10^{5}$ ) and a value of $\delta=-0.2$, the equation for $R_{\mathrm{VV}}$ is valid only in the angular range $\theta \leq 10^{\circ}$, when $\lambda=$ $4090 \AA$. This is almost inaccesible to experimental determination, with the instruments now available. We have to conclude that the approximate equations are not adequate to predict the intensities of polarized light scattering of macromolecules having a rigid structure which can be suitably represented by long anisotropic rods, such as is the case with DNA fragments. Unfortunately, the exact equations of Nagai, which are valid for any angular range (eq 37-39 of ref 1), are too complex to be applied to continuous models and they would spend too much computing time if applied to discontinuous models, such as our double helical one.

The relationship between such a double helical model and the equivalent rigid rod can be deduced from the data shown on Table I. We see on the Table that for a double helix of $A=8 \AA$ (which is the value obtained by comparison with $\mathrm{X}$-ray scattering diagrams $\mathrm{s}^{12}$ ), and principal polarizabilities 90,40 , and $20 \AA^{3},{ }^{13}$ the magnitudes which determine the anisotropic scattering for the two models converge very fast as $N$ grows. For a DNA fragment of $L=1500 \AA$ (such as the one considered in the preceding paragraph), $N=$ 884 , and the difference between the two models is vanishingly small, according to Table $I$ (the value of the anisotropy for DNA is even smaller than the one used to construct Table I, so that the figures tabulated are in excess of the actual ones).

In addition to this, since the distances characteristic of the double helical structure (radius, pitch) are much shorter than $\lambda$, the influence of the cross-section on the isotropic light scattering should be very small also. In fact, the differences in $P(\theta)$ between the rigid rod and the double helix are irrelevant, as we have demonstrated elsewhere. ${ }^{16}$

In summary, the technique of polarized light scattering is not sensitive to the double-helical structure of DNA, at least in the low angle region where Nagai's approximate theory holds. The cross-sectional structure should make a more important contribution to the polarized light scattering in the region of high angles where the exact equations of Nagai are required.

The scattering of unpolarized light is a conventional technique in polymer characterization. Macromolecular parameters are usually obtained by analysis of the unpolarized light-scattering data according to the method of Zimm. In a Zimm plot, the linear extrapolation to zero angle implies a power series expansion, up to terms of order $h^{2}$, not only of the anisotropic contribution to $R$, but also of the isotropic one. We have already seen that the range of validity of such an expansion of the anisotropic part of polarized light is extremely limited, in the case of rigid DNA. The question remains of how good the Zimm plot is for the unpolarized light. We tackle now this problem by studying the Zimm plot of the rigid rod model. We have just seen that, in the low angle region where the expanded formulas are expected to hold, the configuration of rigid DNA is adequately represented by the rod model.

The series expansions of Horn, et al.'s equations, up to terms in $h^{2}$, yield:

$$
\begin{aligned}
R_{\mathrm{VV}} / K c M= & \left(\frac{5+4 \delta^{2}}{5}\right) \\
& \times\left\{1-\frac{35+20 \delta^{2}-28 \delta}{252\left(5+4 \delta^{2}\right)}(h L)^{2}+\cdots\right\} \\
R_{\mathrm{VH}} / K c M= & \left(\frac{3 \delta^{2}}{5}\right)\left\{1-\frac{(h L)^{2}}{28}+\cdots\right\} \\
R_{\mathrm{HH}} / K c M= & \left(\frac{5+4 \delta^{2}}{5}\right)\left\{1-\left[\frac{35+20 \delta^{2}-28 \delta}{252\left(5+4 \delta^{2}\right)}\right.\right.
\end{aligned}
$$




$$
\left.\left.+\left(\frac{\lambda}{4 \pi L}\right)\left(\frac{20+4 \delta^{2}}{5+4 \delta^{2}}\right)\right](h L)^{2}+\cdots\right\}
$$

If incident and scattered light are both unpolarized, the corresponding Rayleigh ratio, $R_{\mathrm{uu}}$, is

$$
R_{\mathrm{uu}}=R_{\mathrm{Vv}}+2 R_{\mathrm{VH}}+R_{\mathrm{HH}}
$$

We calculated $R_{\mathrm{uu}}$ with the exact equations of Horn, et al., and with the expanded ones (eq $28-30)$ and compared both results. They are shown on Figure 2, plotted in the usual way as $K c M\left(1+\cos ^{2} \theta\right) / R_{\mathrm{uu}}$ vs. $\sin ^{2}(\theta / 2)$. The continuous curves represent the exact results and the discontinuous straight lines the linear expansions given by eq $28-30$. The results have been calculated for a value of $L / \lambda$ typical of DNA fragments $(L=1500 \AA, \lambda=4000 \AA$ ).

As we can see, the range for which the initial tangents and the curves coincide is extremely short and decreases as the anisotropy, $\delta$, grows. This means that the Zimm plot for this kind of macromolecule should deviate considerably from linearity. Instead of the Zimm plot, some authors ${ }^{17}$ prefer to determine $M$ and $L$ from the asymptotic behavior of $P(\theta)$, which is propor-

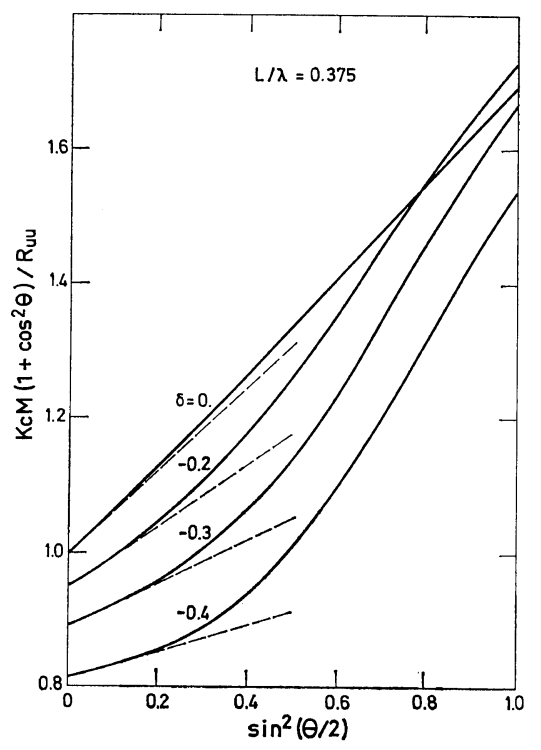

Figure 2. Rayleigh ratios for unpolarized light scattering from a rigid rod: —, exact results calculated according to Horn, et al.5,6; ----, linear extrapolation of the Zimm-plot type. tional to $h$. As we have shown elsewhere, ${ }^{16}$ this may not be a good procedure because the $L$ and $\lambda$ usually involved yield $h L$ values which are not large enough for the asymptotic behavior to be reached. For this reason, we believe that the determination of $M$ and $L$ for anisotropic rigid macromolecules from measurements of $P(\theta)$ should avoid any limiting law, valid either for low or high angles. Instead, it will be more correct to fit the experimental data to the exact theoretical results of the idealized rod model.

An alternative to the use of integrated intensities $(P(\theta))$ for the study of anisotropic rigid macromolecules are the advances made by Pecora, ${ }^{18}$ Tagami, ${ }^{19}$ and Maeda and Saito ${ }^{20}$ concerning the interpretation of the quasi-elastic light scattering from rod models. The theories developed by these authors relate the translational and rotational diffusion coefficients of the rod to the spectrum of polarized light scattering. However, no experiments on rigid fragments of DNA are yet available for the application of these theories, as Berne and Pecora ${ }^{21}$ have pointed out.

Acknowledgement. J.G.T. acknowledges the award of a Fellowship by the Ministerio de Educación y Ciencia for the completion of this work.

\section{APPENDIX}

The numerical coefficients appearing in eq 16 and 17 are as follows:

$$
\begin{array}{rrrrrr} 
& x_{1} & x_{2} & x_{3} & x_{4} & x_{5} \\
f_{3} & -7 & 6 & -4 & - & - \\
f_{4} & - & - & - & 8 & -3 \\
f_{5} & - & - & - & -3 & 9 \\
f_{6} & - & - & - & 5 & 6 \\
f_{7} & 21 & -4 & 12 & - & - \\
f_{8} & 7 & 3 & -2 & - & -
\end{array}
$$

\section{REFERENCES}

1. K. Nagai, Polym. J., 3, 67 (1972).

2. K. Nagai, ibid., 3, 563 (1972).

3. H. Utiyama and M. Kurata, Bull. Inst. Chem. Res. Kyoto Univ., 42, 128 (1964).

4. H. Utiyama, J. Phys. Chem., 69, 4138 (1965).

5. P. Horn, H. Benoit, and G. Oster, J. Chim. Phys., 48, 530 (1951).

6. P. Horn, Ann. Phys. (Paris), 10, 386 (1955). 
7. G. D. Patterson, Macromolecules, 7, 220 (1974).

8. P. Horn and H. Benoit, J. Polym. Sci., 10, 29 (1953).

9. Y. Mauss, J. Chambron, N. Daune, and H. Benoit, J. Mol. Biol., 27, 579 (1967).

10. G. Weill, C. Hornick, and S. Stoylov, J. Chim. Phys., 65, 182 (1968).

11. J. Strassburger, Studia Biophys., 45, 121 (1974).

12. J. García de la Torre and A. Horta, J. Phys. Chem., 80, 2028 (1976).

13. Regarding polarizabilities, see ref 6 and 10 .

14. V. N. Tsvetkov in "Newer Methods of Polymer Characterization,” B. Ke, Ed., Interscience Pub- lishers, Inc., New York, N.Y., 1963, Chapter 14.

15. J. L. Sarquis and R.E. Harrington, J. Phys. Chem., 73, 1685 (1969).

16. J. García de la Torre, Doctoral Thesis, Universidad Complutense, Madrid (1976).

17. H. Benoit, J. Chim. Phys., 65, 23 (1968).

18. R. Pecora, J. Chem. Phys., 49, 1036 (1968).

19. Y. Tagami, J. Chem. Phys., 54, 4990 (1971).

20. H. Maeda and N. Saito, Polym. J., 4309 (1973).

21. B. J. Berne and R. Pecora, "Dynamic Light Scattering," Wiley \& Sons, Inc., New York, N.Y., 1976, p 192. 